\title{
INVESTIGATION OF WAVEGUIDE ANTENNA ARRAYS WITH LAYERED DIELECTRICS
}

\author{
V.M. Morozov, V.I. Magro* \\ Oles Honchar Dnipro National University, Dnipro, Ukraine \\ *e-mail:magrov@i.ua
}

\begin{abstract}
A general methodology for calculating antenna arrays with layered dielectric is considered. To demonstrate the proposed methodology, we consider the solution of the problem of radiation of linear waveguide array. Plane-parallel waveguides have a finite wall thickness. Antenna array scans in $\mathbf{E}$ and $\mathrm{H}$ planes. This problem is solved using Fredholm integral equations of the second kind. To solve the problem, the entire domain of the electromagnetic field is divided into partial intersecting regions An example of constructing Green's functions of selected partial regions with allowance for their dielectric filling is considered. The formulation of the boundary-value problem for the antenna array in the form of Fredholm integral equations of the second kind using the technique of dividing the determination domain into partial regions with Green's functions allows us to solve the following problem: calculations of the antenna array with dielectric plugs in the waveguides and with a dielectric coating over the antenna array. This technique allows optimization of dielectric parameters with the aim of aperture matching of the antenna array. The calculation results of the dependence of the reflection coefficient on the phase shift between waveguides in the H-plane are presented. The calculations are performed for different values of the dielectric constant of the plugs in the waveguides and the dielectric constant of the dielectric layer above the antenna array. The influence of the geometric dimensions of the plugs in the waveguides and the dielectric coating above the array is studied.
\end{abstract}

Keywords: antenna array, integral equation, Green's function, dielectric plug, reflection coefficient.

Received 05.11.2019; Received in revised form 11.12.2019; Accepted 23.12.2019

\section{Introduction}

When designing a waveguide antenna array, it becomes necessary to match the characteristic of impedance of the open end of the waveguide with the wave impedance of the free space. Technical methods for implementing this problem may be different. This can be changes in the geometric dimensions of the radiator near the aperture [1 - 4] or use of dielectrics [5]. In the antenna array, the dielectric can be placed in the plane of the aperture or dielectric plugs in the waveguides can be used. In addition to the function of matching the antenna array with free space, dielectric materials perform protective functions in antenna array. Therefore, the study of the effect of multilayer dielectric filling in the antenna array is an important problem. When solving this problem numerically, it becomes necessary to construct Green's functions [6].

\section{Formulation of the problems}

Let us consider a study of the effect of multilayer magneto dielectrics in an antenna array on the example of a linear waveguide antenna array model from plane-parallel waveguides with a finite wall thickness. The antenna array scans in the E- and H-planes. We will use the integral equation method for analysis. The whole region of field definition can be conditionally divided into overlapping regions (Fig. 1).

In contrast to [7], where the concept of "secondary waves" and the Green function in the resonator representation were used to take into account the magneto-dielectric filling, we consider the solution of this problem based on the Green functions of the selected regions taking into account the magneto-dielectric filling.

As an example, we construct the Green functions of the Dirichlet problem in a "sourcelike" form for distinguished overlapping regions: the "waveguide channel", which is extended to infinity, the Floquet channel in the presence of one magneto dielectric layer.

The system of integral representations for the full fields of the selected regions has the form: 


$$
\begin{gathered}
E^{I}(x, z)=E_{e x c}(x, z)+\sum_{i=1}^{2}(-1)^{i+1}\left[\int_{0}^{\tau} E^{I I}\left((-1)^{i} \frac{a}{2}, z^{\prime}\right) G^{I[2](3)}\left(x, z ;(-1)^{i} \frac{a}{2}, z^{\prime}\right) d z^{\prime}\right. \\
\left.+\int_{\tau}^{\infty} E^{I I}\left((-1)^{i} \frac{a}{2}, z^{\prime}\right) G^{I[3](3)}\left(x, z ;(-1)^{i} \frac{a}{2}, z^{\prime}\right) d z^{\prime}\right] ; \\
E^{I I}(x, z)=\int_{-a / 2}^{a / 2} E^{I}\left(x^{\prime}, 0\right) G^{I I(4)}\left(x, z ; x^{\prime}, 0\right) .
\end{gathered}
$$

Here, the second superscript in the Green's function of the first subdomain denotes the number of the subdomain, into which the entire region is divided, depending on the values of the dielectric constant and magnetic permeability.

In this case, $E_{\text {exc }}(x, z)$ includes an incident wave of unit amplitude and a reflected wave with a complex amplitude coefficient.

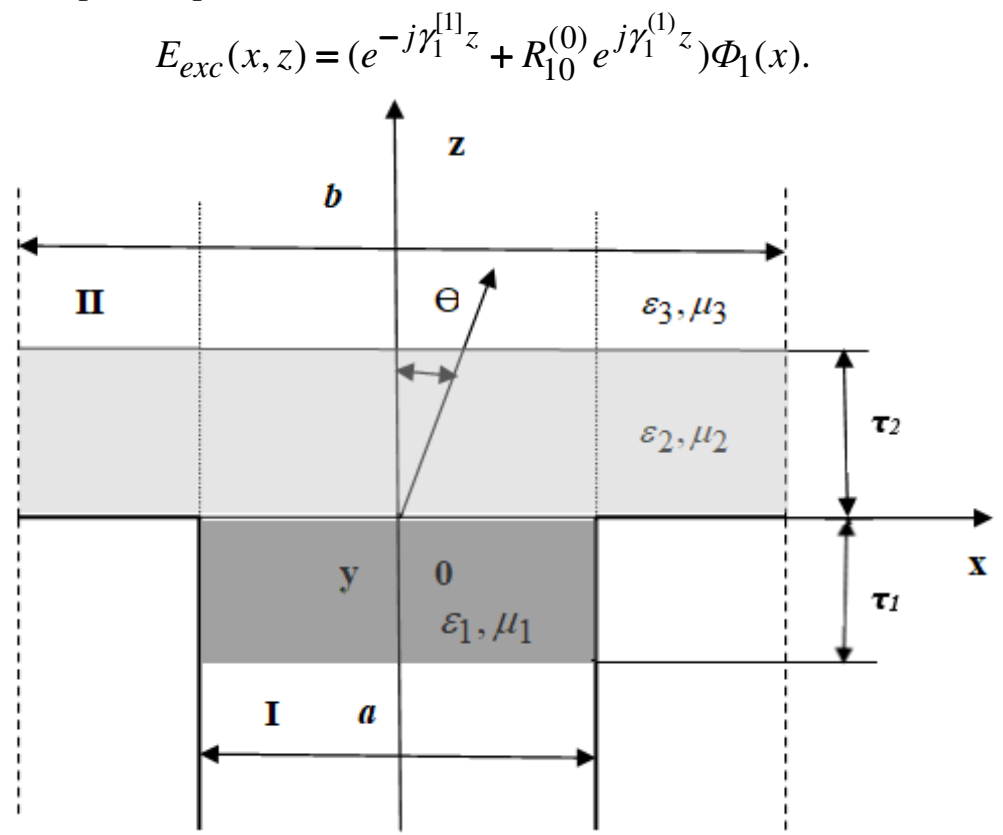

Fig. 1. Selection of overlapping regions for a linear antenna array.

From the set of equations (1) it is seen that the source points are in the plane $z^{\prime}=0$. In the first equation, $z^{\prime}$ varies from zero to infinity. Here, the source points are introduced from the $z=0$ plane into the first region. In the second equation $z^{\prime}=-0$; therefore, the source points are in the plane of occurrence of irregularity. Therefore, when constructing the Green's function of the first region, it is necessary to locate the source points in region I where the parameters of magneto dielectric are $\varepsilon_{1}, \mu_{1}\left(z^{\prime}=-0\right)$. If there are plugs in the waveguides, it is necessary to place the source points in the plugs closest to the OX axis. When constructing the Green function of the second region, it is necessary to place the source points in the first layer of the antenna array coverage from the OX axis $\left(z^{\prime}=+0\right)$.

112 
In the second equation of set (1), the Green function of the second region is written without indicating the number of the subdomain ( 2 or 3$)$, which is determined by substituting this equation into the first equation depending on the location of the integration points.

Let us consider the construction of the Green's function of the "wave channel" taking into account the magneto-dielectric filling in the "source-like" form. For the considered problem of full magneto-dielectric filling along the cross-section of the waveguide, the construction of the Green function in a "source-like" form

$$
G^{I}\left(x, z ; x^{\prime}, z^{\prime}\right)=\sum_{q=1}^{\infty} \Phi_{q}(x) \Phi_{q}\left(x^{\prime}\right) f_{q}\left(z, z^{\prime}\right)
$$

is reduced to finding a longitudinal waveguide function, which depends on the location of the observation point $z$. For an infinite regular waveguide, the function $f_{q}\left(z, z^{\prime}\right)$ satisfies equation

$$
\left(\frac{\partial^{2}}{\partial z^{2}}-\gamma_{q}^{2}\right) f_{q}\left(z, z^{\prime}\right)=-\delta\left(z-z^{\prime}\right)
$$

and has the following form: $f_{q}^{\text {waveguide }}\left(z, z^{\prime}\right)=\frac{e^{-\gamma_{q}\left|z-z^{\prime}\right|}}{2 \gamma_{q}}$. This problem consists in considering the incidence of a plane electromagnetic wave, which is excited by the plane $z^{\prime}$ and is defined by the function $f_{q}^{\text {waveguide }}\left(z, z^{\prime}\right)$ (external source) on the system of magneto dielectric layers with various parameters (Fig. 2).

Let us consider the construction of the Green function of the "Floquet channel" taking into account the magneto-dielectric filling in the "source-like" form. In this case, the construction of the Green function of the second region is similar to the construction of the Green function of the "wave channel", but has its own characteristics. They consist in the fact that the longitudinal wave function is selected for a plane-layered half-space, which is limited at one side by a metal screen (Fig. 3).

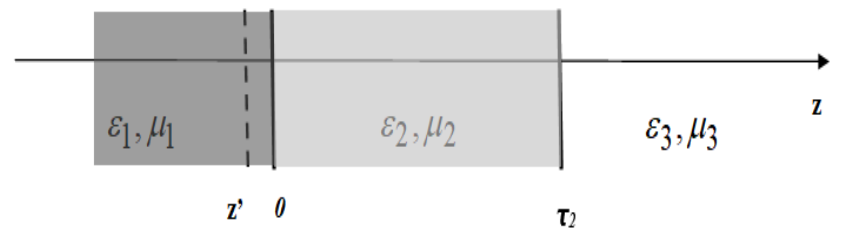

Fig. 2. The selection of subdomains in the waveguide channel.

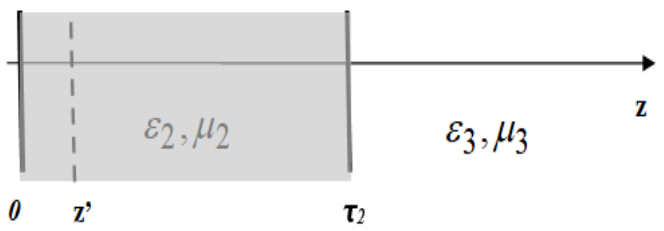

Fig. 3. The selection of subdomains in the "Floquet channel". 

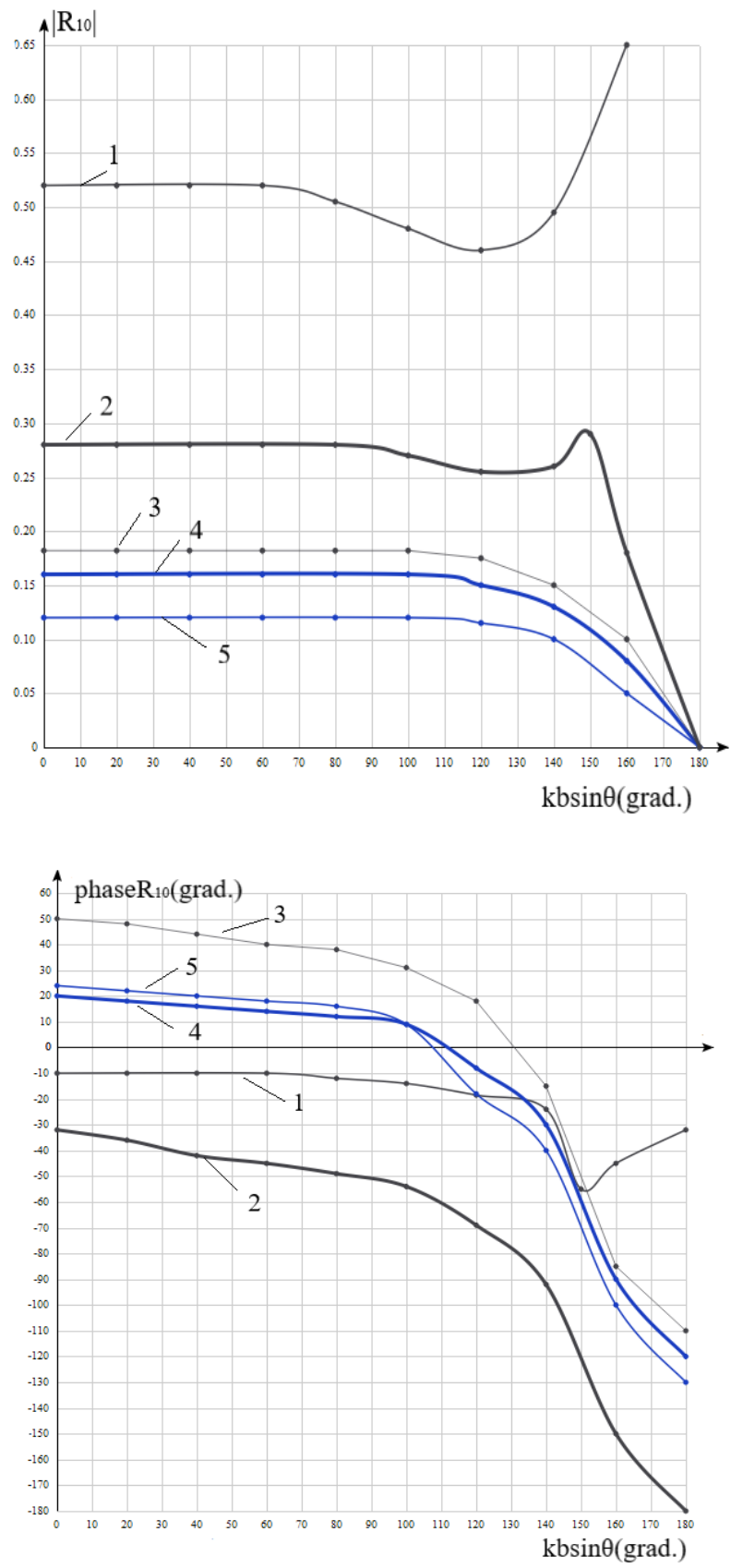

Fig. 4. The dependence of the reflection coefficient $R_{10}$ on the radiation angle $\Theta$ in the H-plane $(a / \lambda=b / \lambda=0.5714)$ in the presence of a dielectric layer in the aperture ( $\varepsilon_{2}=3.0625 ; \tau_{2} / \lambda_{\varepsilon}=0.125$ ) and dielectric plugs in the waveguides $\left(1-\varepsilon_{1}=1 ; \tau_{1} / \lambda_{\varepsilon}=0 ; 2-\varepsilon_{1}=2 ; \tau_{1} / \lambda_{\varepsilon}=0.5 ; 3-\varepsilon_{1}=2 ; \tau_{1} / \lambda_{\varepsilon}=0.125\right.$; $\left.4-\varepsilon_{1}=2 ; \tau_{1} / \lambda_{\varepsilon}=0.375 ; 5-\varepsilon_{1}=2 ; \tau_{1} / \lambda_{\varepsilon}=0.250\right)$. 
There are two ways to find the function $F_{q}^{\text {waveguide }}\left(z, z^{\prime}\right)$. The first method is to consider the incidence of a plane electromagnetic wave, which is excited by the plane $z^{\prime}$, at the borderline. In this case, the Green function of a regular waveguide is used as an external source. In addition, the condition of equality to zero is introduced for the value $z=0$ of the longitudinal waveguide function of the first subregion.

The second method is based on the use of the mirror image method. This leads to the introduction of an additional source, which is located on the other side of the metal screen.

\section{Results and discussion}

The solution of the boundary problem for the antenna array leads to Fredholm integral equations of the second kind. In this case, the conditional division of the field definition domain into partial overlapping regions with the Green functions of layered media is used. This allows us to solve the following problems: calculation of the antenna array in the presence of a dielectric in the aperture plane and dielectric inserts in the waveguides; optimization of magneto-dielectric filling values to match the antenna array with free space. A numerical study of the dependence of the reflection coefficient on the scanning angle was carried out for the dielectric constant of the plugs in the waveguides $\varepsilon_{\text {plags }}=2$. In this case, the thickness of the dielectric layer in the aperture $\tau / \lambda_{\varepsilon}$ changed from 0.125 to 0.5 . For such values of the dielectric layer thickness in the aperture, there is one spatial harmonic (-1) of the type of surface wave in the range of scanning angles $0 \leq k b \sin \theta \leq 2 \pi(1-b) / \lambda$. The wavelength value was chosen close to the cutoff wavelength. The thickness of the dielectric layer in the aperture is selected based on the condition of the absence of resonances of surface waves. A numerical study of the dependence of the reflection coefficient $R_{10}$ on the radiation angle $\Theta$ in the $\mathrm{H}$ plane in the presence of a dielectric layer in the aperture and dielectric plugs in the waveguides was performed (Fig. 4). The combined use of dielectric plugs in the waveguides and the dielectric layer in the aperture allows matching the antenna array with free space (curve 5 in Fig. 4). In this case, the dependence of the reflection coefficient modulus on the scanning angle has the form of an almost flat curve.

\section{Conclusions}

The numerical analysis shows that $R_{10}^{(0)}$ (the reflection coefficient from the layered filling of region I) coincides with the reflection coefficient of the grating with zero wall thickness of the waveguides when radiation at an angle $\theta$, which is determined from the condition $k b \sin \theta=\pi$, i.e. at the maximum angle, at which numerical modeling of the antenna array is still possible. This is due to the pairwise interference of spatial harmonics. As a result of this process, the spectrum of spatial harmonics in the "Floquet channels" is transformed into a waveguide spectrum. The zero and first negative spatial harmonics are transformed into the wave $H_{10}$, the first and second negative harmonics are transformed into the wave $H_{30}$. Since the transverse geometric dimensions of the "wave channel" and "Floquet channel" coincide $(a=b)$, only the wave $H_{10}$ propagates in the radiation region. 


\section{References}

1. Christie, L. Mode matching method for the analysis of cascaded discontinuities in a rectangular waveguide / L. Christie, P. Mondal // Proceedings of the 6th International Conference on Advances in Computing and Communications. - 2016. -Vol. 93. - P. 251 -258 .

2. Gnatyuk, M.A. An integral equation technique for the analysis of phased array antenna with matching step discontinuities/ M.A. Gnatyuk, V.M. Morozov // Journal of Physics and Electronics.- 2018.- Vol. 26(2).- P. 101 - 106.

3. Magro, V.I. Study of a matching device for finite linear waveguide phase antenna arrays / V.I. Magro, V.M. Morozov // Izvestiya Vysshikh Uchebnykh Zavedenii. Radioelektronika. - 1997. - № 7. - S. 30 - 34.

4. Magro, V.I. Mathematical modeling of the wave diffraction on open periodic structures / V.I. Magro, V.M. Morozov // Proc. of the Second International Conference "Antenna theory and techniques". - Kyiv (Ukraine). -1997. - P. 262 - 263.

5. Amitay, N. Theory and analysis of phased array antennas / N. Amitay, V. Galindo, C. Wu. - New York: Wiley-Interscience, 1972. - 462 p.

6. Prokhoda, I.G. Tenzornye funktsii Grina i ikh primemenie v electrodinamike SVCh / I.G. Prokhoda, S.G. Dmitriuk, V.M. Morozov. - Dneproperovsk: DGU, 1985. $64 \mathrm{p}$.

7. Onufriyenko, V.M. Raschet volnovodnykh transformatorov $\mathrm{s}$ chastichnym dielektricheskim zapolneniyem / V.M. Onufriyenko, I.G. Prokhoda // Radiotekhnika i elektronika. - 1976. - T. 21. - № 6. -S. 1318 - 1320 